\title{
2.8 Unique Patterns of Elastin Degradation in Ascending Aortic Aneurysms in Bicuspid Aortic Valve Patients
}

\author{
Ya Hua Chim ${ }^{1}$, Hannah Davies ${ }^{1}$, Omar Nawaytou $^{2}$, Mark Field ${ }^{2}$, Jillian Madine ${ }^{1}$, Riaz Akhtar $^{1,}$ \\ ${ }^{1}$ University of Liverpool, Liverpool, UK \\ ${ }^{2}$ Liverpool Heart and Chest Hospital, Liverpool, UK
}

\section{ABSTRACT}

Background: Bicuspid aortic valve patients (BAV) are associated with increased risk of ascending aortic aneurysms. However, is unclear whether matrix degradation varies in different ascending aneurysm aetiologies.

Aim: To determine whether the micromechanical and biochemical properties elastin microstructure in BAV-A exhibit unique properties as compared to idiopathic degenerative aneurysm (DA) for the same aortic size.

Methods: Aortic tissue was obtained from patients undergoing aneurysmal repair surgery (BAV-A; $n=15$ and DA; $n=15$ ) Coronary artery by-pass graft punch biopsies served as controls (CABG; $n=9)$. The elastic modulus $(\mathrm{E})$ was measured with nanoindentation for the medial layer. Glycosaminoglycan (GAG), collagen and elastin levels were measured using biochemical assays. Verhoeff Van Gieson-stained sections were imaged for elastin microstructural quantification.

Results: BAV-A had approximately 20\% higher E relative to control and DA. There was no significance between DA and controls. Collagen level of BAV-A $(36.9 \pm 7.4 \mu \mathrm{g} / \mathrm{mg})$ and DA $(49.9 \pm 10.9 \mu \mathrm{g} / \mathrm{mg})$ was higher compared to the control $(30.2 \pm 13.1 \mu \mathrm{g} / \mathrm{mg})$. GAG and elastin levels were not significant between the groups. Elastin segments were uniform in controls. Aneurysmal tissues had loss of segments close to the intima and adventitia layers. Although BAV-A and DA had more elastin segments compacted in the media, elastin segments were highly fragmented in DA.

Conclusion: BAV-A has increased stiffness within the aortic wall relative to DA and control tissue. The spatial distribution of elastin demonstrated a unique profile of matrix degradation for BAV-A. The findings of this work are important for the development of future clinical treatment of BAV-A treatment.

(c) 2019 Association for Research into Arterial Structure and Physiology. Publishing services by Atlantis Press International B.V. This is an open access article distributed under the CC BY-NC 4.0 license (http://creativecommons.org/licenses/by-nc/4.0/). 